\title{
Le Radeau des Cimes au Parc national de Masoala (Madagascar). Première partie : éléments pour un inven- taire des Lépidoptères
}

\author{
Henri-Pierre Aberlenc ${ }^{1}$, Lanto Andriamampianina ${ }^{2}$, Éric Faure ${ }^{3}$, \\ David C. Lees ${ }^{4}$, Joël Minet ${ }^{5}$, Laurence Ollivier ${ }^{6}$, Casimir \\ Rafamantanantsoa ${ }^{7}$, Maminirina Randrianandrasana ${ }^{8}$ et \\ Andrianantenaina Razafindrakotomamonjy ${ }^{9}$
}

1 UMR Cbgp, CIRAD, TA A-55 / L, Campus international de Baillarguet - CSIRO, F-34398 Montpellier Cedex 5 - henri-pierre.aberlenc@cirad.fr

2 Wildlife Conservation Society, Villa Ifanomezantsoa, Face II A 78 D, Soavimbahoaka, B.P. 8500, Antananarivo 101, Madagascar - lanto@wcs.org

3 E.R. Biodiversité et environnement, case 5, Université de Provence, place Victor Hugo, F-13331 Marseille Cedex 3, France - eric.faure@up.univ-mrs.fr

4 Department of Entomology, The Natural History Museum, Cromwell Road, London SW7 5BD, United Kingdom - dclees@gmail.com

5 Muséum national d'histoire naturelle, Département systématique \& évolution, USM 602, Case Postale ${ }^{\circ}$ 50 (Entomologie), F-75231 Paris Cedex 05, France - minet@mnhn.fr

6 CIRAD, TA TA A-55 / L, Campus international de Baillarguet - CSIRO, F-34398 Montpellier Cedex 5 - laurence.ollivier@cirad.fr

7 Laboratoire d'Entomologie, Parc botanique et zoologique de Tsimbazaza, B.P. 4096, Antananarivo 101, Madagascar

8 Department of Entomology, University of Illinois, 320 Morrill Hall, 505 S. Goodwin, 61801 Urbana, Illinois, U.S.A. - randrianandrasanamaminirina@yahoo.fr

9 Laboratoire d'entomologie, Faculté des sciences, Université d'Antananarivo, B.P. 906, Antananarivo 101, Madagascar - razafindrakotomamonjy@yahoo.fr

Résumé. - Grâce à l'expédition Radeau des Cimes 2001, de nombreuses espèces de Lépidoptères peuvent être rajoutées aux inventaires faunistiques de la presqu'île de Masoala, encore très incomplets. Des papillons et quelques chenilles ont été collectés pendant cinq semaines (principalement en novembre) dans la région de Tampolo, mais aussi dans celle d'Andranobe, au sud d'Ambanizana. Un certain nombre de chenilles ont été élevées avec succès, notamment celle d'une noctuelle. apparemment non décrite (Herminiinae) et la chenille jusque-là inconnue du Sphingidae Rhagastis lambertoni (Clark). À partir de nids d'un Hypsoides sp. (Notodontidae Thaumetopoeinae ; espèce peut-être nouvelle), divers insectes ont été obtenus, dont un Cosmopterigidae et un Phycitinae (Pyralidae) vraisemblablement des espèces nouvelles pour la science. Au moins 23 espèces de Bombycoïdes (dont le Sphingidae Hippotion melichari Haxaire) ont pu être cupturées - en octobre-novembre 2001 - dans la région de Tampolo. En comparant l'efficacité de trois pièges lumineux similaires, mais installés, l'un sur le Radeau des Cimes, les deux autres sous celui-ci, dans le sous-bois, on peut avancer l'hypothèse selon laquelle la plupart des Sphinx nocturnes tendraient à voler au-dessus de la canopée. Une première liste des Sphingidae de Masoala est proposée. Parmi les Rhopalocères, 65 espèces de Rhopalocères ont été distinctement aperçues (p. ex. Smerina manoro (Ward) - Nymphalidae) ou capturées. Trois Hesperiidae sont signalés pour la première fois de Masoala : Perrotia howa (Mabille), P. silvestralis (Viette) et Borbo ratek (Boisduval).

Mots-clés. - Lépidoptères, premiers états, Sphingidae, Radeau des Cimes, Masoala.

Accepté pour publication le 15 mars 2007. 


\section{The Canopy Raft in the Masoala National Park (Madagascar). 1. - Towards a Lepidoptera survey}

Summary. - Thanks to the 2001 "Canopy Raft" expedition, many new records can be considered for the Lepidoptera fauna of the Masoala Peninsula, a still poorly investigated region of Madagascar. Moths, butterflies and a few caterpillars were collected over a five week period (mainly in November) in the area of Tampolo, but also near Andranobe, south of Ambanizana. A number of caterpillars have been bred successfully, in particular that of an apparently new herminiine species (Noctuidae) and the previously unknown larva of Rhagastis lambertoni (Clark) (Sphingidae). Several insect species were obtained from the nests of a possibly new species of Hypsoides (Notodontidae: Thaumetopoeinae). Among them are a Cosmopterigidae and a phycitine Pyralidae, both of which are likely to represent new species. At least 23 species of Bombycoidea (e.g. Hippotion melichari Haxaire - Sphingidae) were caught in october-november 2001 in the Tampolo area. Through a comparison between the efficiency of a light trap put on the canopy raft and that of two similar ones placed under the raft, in rainforest undergrowth. it would seem that most nocturnal hawk-moths tend to fly above canopy level. A checklist of the Sphingidae of Masoala can be proposed for the first time. 65 species of Rhopalocera were indisputably seen (e.g. Smerina manoro (Ward) - Nymphalidae) or collected. Three hesperiids are newly reported for Masoala: Perrotia howa (Mabille), P. silvestralis (Viette) and Borbo ratek (Boisduval).

Keywords. - Lepidoptera, early stages, Sphingidae, Canopy Raft, Masoala.

\section{INTRODUCTION}

Contrairement à la pluparı des régions de Madagascar, la presqu'île de Masoala n'a guère été explorée par les entomologistes avant le début des années 1990 (à l'exception des environs de Hiaraka (15²9'S, 4954'E) : VIETTE, 1969). Il s'agit pourtant d'une zone fort intéressante, car encore largement recouverte de forêt primaire, y compris - à l'ouest - le long du littoral de la baie d'Antongil. Il faut d'ailleurs souligner que la pluvisylve de basse altitude a presque entièrement disparu des autres endroits de la côte est de Madagascar. On ne peut donc que se féliciter du statut de Parc national récemment accordé à la majeure partie de la presqu'île Masoala (KREMEN, 2003).

En 1993-1994, divers endroits de cette presqu'île ont été visités par des entomologistes, en particulier pour arriver à dresser un premier inventaire de ses Lépidoptères Rhopalocères (KREMEN et al., 2001). L'un d'entre nous (DCL) faisait partie de l'équipe et avait ainsi pu récolter de nombreuses espèces de papillons - diurnes et nocturnes à Masoala, notamment au sud d'Ambanizana, dans la forêt entourant Andranobe (1540'54"S, 4957'26”'E). L'expédition Radeau des Cimes 2001 nous a permis d'étudier les Lépidoptères d'une zone situéc lćgc̀rcment plus au sud, à savoir Tampolo et ses environs immédiats.

Le dirigeable à air chaud (48 mètres de long, $\left.7500 \mathrm{~m}^{3}\right)$ déposa sur la canopée de la forêt pluviale la dernière version du Radeau des Cimes, le Bretzel, une plate-forme pneumatique trilobée de $400 \mathrm{~m}^{2}$ servant de site d'observation, de prélèvement et d'expérimentation pour les équipes scientifiques. L'accès à la canopée était également rendu possible par deux Icos (structures icosaédriques rigides assemblées sur des émergents, véritables « maisons dans les arbres »), par la Bulle des Cimes à hélium (ballon auquel 
une personne est suspendue, pouvant ainsi marcher sur la canopée le long d'une cordeguide mise en place à l'aide du dirigeable) et par la Luge des Cimes (plate-forme suspendue sous le dirigeable, pour faire des prélèvements itinérants dans la canopée).

Tampolo est plus un lieu-dit qu'un véritable village : notre campement et son laboratoire temporaire $\left(15^{\circ} 43^{\prime} 48^{\prime \prime} \mathrm{S}, 49^{\circ} 57^{\prime} 36^{\prime \prime} \mathrm{E}\right)$ se trouvaient sur l'emplacement d'une plantation de canneliers, un peu au nord de la Pointe Tampolo, à environ $6 \mathrm{~km}$ au sud d'Andranobe (à vol d'oiseau), c'est-à-dire approximativement au milieu de la côte occidentale de la presqu'île Masoala. Nous avons essentiellement prospecté la région comprise entre Tampolo (alt. $<10 \mathrm{~m}$ ) et le Radeau des Cimes (alt. $150 \mathrm{~m} ; 15^{\circ} 42^{\prime} 46^{\prime \prime} \mathrm{S}$, $49^{\circ} 58^{\prime} 16^{\prime \prime} \mathrm{E}$ ), mais aussi quelques points situés au nord-est par rapport à ce dernier. En outre, plusieurs d'entre nous se sont rendus à Andranobe, soit (CR ; MR) pour y piéger des Rhopalocères, soit (DCL ; JM) pour effectuer, en compagnie de collègues botanistes, une excursion jusqu'à Ambatoavo (alt. $600 \mathrm{~m} ; 15^{\circ} 40^{\prime} 13^{\prime \prime} \mathrm{S}, 49^{\circ} 58^{\prime} 40^{\prime \prime} \mathrm{E}$ ), voire jusqu'à Be Dinta (alt. $550 \mathrm{~m} ; 15^{\circ} 38^{\prime} 30^{\prime \prime} \mathrm{S}, 49^{\circ} 57^{\prime} 30^{\prime \prime} \mathrm{E}$ ). Ces deux derniers sites l'un et l'autre en forêt primaire — correspondent à des biotopes passablement différents dans la mesure où Be Dinta est un endroit bien plus humide, essentiellement marécageux.

Nous avons donc mis à profit la mission Radeau des Cimes pour étudier les Lépidoptères de la région de Tampolo-Andranobe (dans la partie occidentale du parc de Masoala), et ce globalement pendant une période de cinq semaines, plus précisément du 24 octobre (arrivée d'HPA) au 27 novembre 2001 (départ de LA, EF et AR). Il s'agissait naturellement de chercher à inventorier les espèces de cette région, mais aussi de tenter certains élevages, les chenilles malgaches restant très mal connues. Nous avions également en vue des recherches sur la biodiversité à l'échelle moléculaire et d'éventuelles observations sur les Lépidoptères pouvant habiter la canopée (à un quelconque stade de leur développement).

\section{MATÉRIEL ET MÉTHODES}

Si les Lépidoptères diurnes ont été le plus souvent capturés de façon classique, à l'aide de filets à papillons, nous avons également utilisé différents types de pièges, notamment pour attirer les espèces nocturnes. Nous avons ainsi presque tous collecté autour des deux ampoules à vapeur de mercure $(2 \times 125 \mathrm{~W})$ du piège non automatique

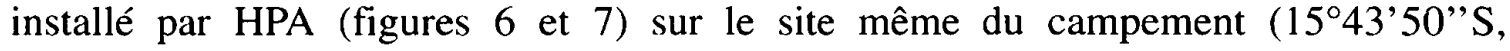
49 57'36'E), piège allumé pratiquement tous les soirs pendant quatre semaines. Des pièges lumineux similaires, quoique moins puissants (à une seule ampoule), nous ont permis d'effectuer des chasses en forêt littorale et en forêt primaire, en particulier sur et sous le Radeau des Cimes (simultanément, afin de pouvoir comparer la faune du sousbois à celle qui survole la canopée). Grâce au Radeau, il a été également possible de suspendre à une même corde - à différentes hauteurs - plusieurs pièges de Malaise (BORROR et al., 1981 : fig. 644 C) et à une autre, de la même façon, trois pièges à Rhopalocères pourvus d'appâts fermentés. Pièges à Rhopalocères et pièges de Malaise ont aussi été utilisés, isolément, en divers autres endroits. Essentiellement constitués d'un long manchon ajouré et d'un plateau porte-appât (Migdoll, 1988 : 22), les premiers ont été généralement garnis de bananes (fermentées dans de la bière) et, contrai- 
rement à ce que suggère leur nom, ont parfois attiré des hétérocères : plus précisément des noctuelles du genre Cyligramma Boisduval, 1833. Les seconds, quant à eux, ont permis la récolte de divers Lépidoptères (microlépidoptères, notamment), mais en alcool à $70^{\circ}$, milieu qui ne facilite guère l'étude de ces fragiles insectes.

Avec la Luge des Cimes, certains d'entre nous ont pu apercevoir des papillons volant peu après l'aurore, en particulier quelques Uraniidae appartenant à une belle espèce endémique : Chrysiridia rhipheus (Drury, 1773) (observation faite par CR). La Bulle des Cimes a été mise à profit pour le ramassage de branches de la canopée portant des chenilles.

En règle générale, les papillons ont été tués dans des bocaux à cyanure, puis conservés à sec, tandis que les chenilles ont été préservées en alcool à $70^{\circ}$. Toutefois, plusieurs chenilles et papillons ont été directement mis en alcool à $95^{\circ}$ ou $100^{\circ}$ en vue de recherches en phylogénie moléculaire.

\section{PREMIERS ÉTATS DE CERTAINS LÉPIDOPTĖRES}

À Tampolo même, sur la plage située à proximité du laboratoire, au moins une dizaine de chenilles (figure 8) d'un grand Saturniidae, Bunaea aslauga Kirby, 1877 ont été trouvées par Gianfranco Curletti et HPA sur Intsia bijuga, un arbre de la famille des Fabaceae. Cette plante-hôte ne semble pas avoir encore été signalée pour $B$. aslauga, espèce dont la chenille est cependant réputée polyphage (GRIVEAUD, 1962 : 46). Les environs de Tampolo ont livré à DCL une chenille fort étrange, manifestement inconnue, mais se rattachant visiblement aux Sphingidae Macroglossinae : elle sera décrite en collaboration avec le Dr Ian J. Kitching, car son élevage réussi permet d'affirmer qu'il s'agit de Rhagastis lambertoni (Clark, 1923) (figure 16), espèce endémique malgache appartenant à un genre essentiellement oriental. C'est également non loin de Tampolo, en forêt primaire, que DCL a réussi à trouver, le 19 novembre, quelques œufs de Chrysiridia rhipheus (Uraniidae) sur un Omphalea (Euphorbiaceae) dont l'espèce resterait à préciser. Ceux-ci ont rapidement donné naissance à de jeunes chenilles : l'une d'elles a été conservée en alcool pour un futur travail sur les premiers états de ce remarquable Uraniidae.

Des nids de processionnaires (figures 1 et 2) ont été découverts par notre ami JeanYves Serein et collectés par HPA au sud de Tampolo (1544'06"'S, 4957'26”E), sur une liane accrochée à un arbre qui n'a malheureusement pas été identifié (peut-être de la famille des Fabaceae, selon les indications fournies par PaULIAN, 1951: 69). Plusieurs imagos des deux sexes (figures 3 à 5) ont émergé des nombreux cocons que renfermaient ces nids, ce qui permet d'apprécier la variabilité de ce Thaumetopoeinae (Notodontidae) : si la plupart des mâles ont les ailes postérieures pratiquement unicolores, quelques-uns montrent une bande brunâtre assez pâle le long du bord externe de ces ailes (figure 5). La détermination de cette espèce s'avère délicate. Il s'agit d'Hypsoides conglomerata (Oberthür, 1923) sensu KIRIAKOFF (1969). Toutefois, la description originale d' $H$. conglomerata ne semble guère correspondre au concept de

Kiriakoff. À titre provisoire, nous baptiserons donc ce taxon Hypsoides sp. Par ailleurs, ces nids abritaient des fourmis (par centaines), des Tachinidae (Diptères parasites dont 
les pupes étaient parfois elles-mêmes hyperparasitées par des microhyménoptères) et deux espèces de microlépidoptères apparemment nouvelles pour la science : un Cosmopterigidae du genre Pyroderces Herrich-Schäffer, 1853, et un Pyralidae de la sous-famille des Phycitinae. Curieusement, en effet, cette pyrale diffère de Metoecis carnifex (Coquerel, 1855), seule phycite signalée - à ce jour - comme hôte de nids d'Hypsoides.

À Tampolo, certains élevages n'ont pu être menés à bien, notamment en raison d'une virose assez contagieuse : c'est ainsi qu'une grande chenille d'Eudocima sp. (= Othreis sp. ; Noctuidae Calpinae) (récolteur : CR) a été retrouvée morte, gisant au milieu d'un liquide rougeâtre, seulement quelques heures après son introduction dans une cage d'élevage modérément aérée ; le phénomène s'est reproduit pour plusieurs autres espèces. En revanche, quelques chenilles d'Herminiinae (Noctuidae) - provenant d'un arbre (Barringtonia, Lecythidaceae) croissant sur une plage située au sud de Tampolo - ont été élevées avec succès et feront l'objet d'une description précise, de même que les deux imagos obtenus en bon état (HPA et JM, en préparation). En effet, l'espèce en question semble nouvelle. De plus, les premiers états des Herminiinae sont, d'une façon générale, encore assez mal connus. Grâce à la Bulle des Cimes, notre collègue Sybille Unsicker a pris dans la canopée trois chenilles d'une autre noctuelle intéressante, puisque faisant visiblement partie des Acronictinae, sous-famille actuellement difficile à délimiter en ce qui concerne Madagascar (LEEs \& MineT, 2003). Ces chenilles n'ont pas été mises en élevage, mais conservées pour une éventuelle identification moléculaire.

Sur la plage ou en forêt littorale au nord de Tampolo, l'un d'entre nous (JM) a trouvé des chenilles de Lymantriidae assez particulières (figures 9 et 10). Elles n'ont pu être élevées, mais appartiennent clairement à deux genres distincts. Contrairement à la chenille photographiée sur Scaevola sericea (Goodeniaceae) (figure 10), l'espèce de la figure 9 a été observée sur des plantes diverses - Aspleniaceae, Melastomataceae... et il n'a pas été possible de déterminer sa véritable plante-hôte. Enfin, nous avons réussi à faire pondre certaines femelles, notamment dans le cas des Arctiidae Phryganopteryx convergens Toulgoët, 1959 (figure 13) et Argina astrea (Drury, 1773) (figure 12). La première espèce se rattache à une tribu endémique de Madagascar (au sein des Lithosiinae - BENDIB \& MINET, [2000]), tribu aux affinités incertaines et dont on ignorait tout des premiers états : l'œuf et le premier stade larvaire pourront désormais être étudiés (JM, en préparation). On notera que les jeunes chenilles ont refusé de consommer de la laitue (naturellement sans pesticides), plante pourtant volontiers acceptée par de nombreux Lithosiinae. La seconde espèce (figure 12), largement répandue dans l'Ancien Monde, a déjà été élevée à plusieurs reprises, généralement sur des Crotalaria (Fabaceae) ; le dernier stade larvaire présente une coloration caractéristique (figure 11 ; voir aussi la planche II, fig. m [et non 1], de DesegaulX DE NoleT, 1984) et a pu être obtenu en France, en remplaçant les feuilles de Crotalaria initialement proposées par des petits pois décongelés (coupés en deux). 


\section{QUELQUES FAMILLES MAL REPRÉSENTÉES À MADAGASCAR}

Dans le cadre de la présente mise au point, il n'est guère possible de dresser l'inventaire de tous les Lépidoptères capturés lors de la mission Radeau des Cimes 2001, notamment parce que plusieurs groupes n'ont pas été vraiment révisés au cours des cinquante dernières années (rappelons que le premier volume de la Faune de Madagascar date de 1956). Nous nous contenterons donc d'attirer l'attention sur certaines familles qui existent à Masoala et ne comprennent que peu d'espèces à Madagascar (familles éventuellement non encore signalées de ce pays).

Pendant longtemps, on a cru que Madagascar ne possédait aucune famille « d'Homoneures ». VIETTE (1990), par exemple, ne mentionne ni Micropterigidae, ni Hepialidae, dans sa liste des «Hétérocères » malgaches. En fait, la première de ces familles est représentée à Madagascar par quelques espèces endémiques, non encore nommées (KRISTENSEN, [1998] ; LeEs \& Minet, 2003). Ces Micropterigidae ont tous été collectés par DCL, le premier spécimen ayant été découvert il y a quelques années, sur une fougère arborescente (Cyathea, Cyatheaceae) d'Ambatoavo. Ce pied de fougère a été retrouvé et réexaminé en 2001, cette fois sans qu'il soit possible d'observer un seul Micropterigidae sous ses feuilles. En outre, aucun Micropterigidae ne semble avoir été capturé à l'aide des pièges Malaise installés en divers endroits. Cela suggère que ces insectes ne volent pas en novembre dans le Parc national de Masoala. Parmi les autres familles de Lépidoptères « inférieurs », c'est-à-dire non Ditrysiens, VIETTE (1990) ne mentionne - pour la faune malgache - qu'un Nepticulidae, un Heliozelidae et trois espèces d'Adelidae. Toutefois, deux de ces dernières sont rangées à tort dans les Adelidae, appartenant en fait aux Ditrysia (LEES \& MiNET, 2003). Un authentique Adelidae a pu être capturé (à Ambatoavo, le 17 novembre) par DCL qui a également trouvé divers Nepticulidae (plusieurs mines ; quelques imagos pris non loin du radeau des cimes, par piégeage lumineux, le 13 novembre).

Au sein des Tineoidea, la famille « primitive » des Eriocottidae n'a été signalée de Madagascar que très récemment (sous-famille des Eriocottinae : LEES \& MINET, 2003). L'un de ses genres - Compsoctena Zeller, 1852 (sous-famille des Compsocteninae) - y est pourtant représenté par plusieurs espèces inédites (JM, données non publiées). L'une de celles-ci a été capturée à l'aide du piège lumineux de Tampolo (le 14 novembre - JM). Au moins deux spécimens d'Alucitidae (Alucitoidea) ont été pris le 10 novembre, par piégeage lumineux (JM ; MR) sous le Radeau des Cimes ; ils n'appartiennent à aucune des deux espèces d'Alucitides déjà décrites de la Grande Île (VIETTE, 1956 ; MINET, 1976). Enfin, notons l'existence à Tampolo (« laboratoire » et piège lumineux - $\mathrm{CR}$ ) du seul Eupterotidae de la faune malgache, Jana palliatella Viette, 1955 (Bombycoidea) et la présence d'un Callidulidae intéressant au nord de Tampolo, en forêt littorale (DCL \& JM), à savoir Griveaudia nigropuncta Viette, 1958 (Calliduloidea).

\section{BOMBYCOÏDES}

La presqu'île Masoala possède trois des quatre familles de Bombycoidea présentes à Madagascar. Il s'agit des Eupterotidae (voir ci-dessus), des Saturniidae et des 
Sphingidae. Au moins 23 espèces de Bombycoïdes ont été capturées à Masoala en octobre-novembre 2001. Parmi celles-ci, une femelle de Saturniidae prise au piège lumineux de Tampolo (le 24 octobre - HPA) s'avère délicate à déterminer : elle appartient au genre Maltagorea Bouyer, 1993, mais ne peut être attribuée à l'espèce $M$. vulpina (Butler, 1882) qu'avec doute selon notre collègue Rodolphe Rougerie, spécialiste de la taxinomie de ce genre. D'ailleurs, jusqu'à présent, $M$. vulpina semblait faire défaut dans les zones de basse altitude.

En ce qui concerne les Sphingidae, le piège lumineux de Tampolo (figures 6 et 7) a permis certaines captures intéressantes : Xanthopan morganii praedicta Rothschild \& Jordan, 1903 (HPA ; CR ; MR) (figure 14), insecte pollinisateur de l'orchidée Angraecum sesquipedale, Temnora engis engis Jordan, 1933 (JM), Daphnis nerii (Linné, 1758) (HPA), le «Sphinx du laurier-rose », Rhagastis lambertoni (Clark, 1923) (HPA) (figure 16) et, surtout, Hippotion melichari Haxaire, 2001 (HPA) (figure 15), espèce de découverte très récente (HAXAIRE, 2001). En comparant les récoltes effectuées le 10 novembre à l'aide de trois pièges lumineux utilisés simultanément l'un posé sur le Radeau des Cimes (DCL), les deux autres installés sous celui-ci, au niveau du sol (JM ; MR) -, on constate que les Sphingidae tendent à voler au-dessus de la canopée, contrairement à divers Hétérocères au vol moins vigoureux. En effet, alors qu'une seule espèce de Sphingidae a été attirée par les pièges lumineux placés dans le sous-bois (Gynoeryx meander (Boisduval, [1875])), sept ont été attrapées grâce au piège situé sur le Radeau (dont $G$. meander).

D'ores et déjà, nous pouvons dresser un premier inventaire des Sphingidae de la presqu'île de Masoala, en tenant compte non seulement de nos récoltes de 2001, mais aussi de celles effectuées par DCL en 1993 et 1994, ainsi que du matériel collecté en 1968, à l'est de Hiaraka, par Paul Griveaud, André Peyrieras et Pierre Viette (VIETTE, 1969 : 23-24). Dans la liste ci-dessous, les lettres mentionnées après le nom d'un taxon donné correspondent à des localités et des dates précisées au paragraphe suivant, « Explication des informations codées ».

\section{SMERINTHINAE}

Batocnema coquerelii coquerelii (Boisduval, [1875]) - c, e

Pseudoclanis grandidieri grandidieri (Mabille, 1879) - b, e, h, l, p

Gynoeryx meander (Boisduval, [1875]) - b, e, g, h, i, j, l, p, r

\section{SPHINGINAE}

Lomocyma oegrapha (Mabille, 1884) - b, c, e, h

Panogena jasmini jasmini (Boisduval, [1875]) - b, c

Panogena lingens lingens (Butler, 1877) - b, p

Xanthopan morganii praedicta Rothschild \& Jordan, 1903 - a, e, p

Coelonia solani solani (Boisduval, 1833) - e, h

Coelonia mauritii (Butler, 1877) - b, c, o, p, r 


$$
-148-
$$

Acherontia atropos (Linnaeus, 1758) - c

\section{MACROGLOSSINAE}

Cephonodes hylas virescens (Wallengren, 1858) - $\mathrm{n}$

Sphingonaepiopsis obscurus (Mabille, 1880) - m

Temnora fumosa peckoveri (Butler, 1877) - o, p, r

Temnora grandidieri (Butler, 1879) - b, $\mathrm{p}$

Temnora engis engis Jordan, 1933 - b, p, r

Temnora argyropeza (Mabille, 1879) - b, p

Temnora nitida Jordan, 1920 - b

Temnora palpalis Rothschild \& Jordan, 1903 - b, p

Nephele comma Hoppfer, 1857 - d, e

Nephele densoi (Keferstein, 1870) - p, r

Nephele oenopion oenopion (Hübner, [1824]) - c, e

Maassenia heydeni heydeni (Saalmüller, 1878) - h

Daphnis nerii (Linnaeus, 1758) - p

Atemnora westermannii (Boisduval, [1875]) - p

Basiothia medea (Fabricius, 1781) - k

Euchloron megaera lacordairei (Boisduval, 1833) - e, p

Theretra orpheus malgassica Clark, 1933 - b, p, r

Hippotion geryon (Boisduval, [1875]) - b, c, p

Hippotion celerio (Linnaeus, 1758) - $\mathrm{k}$

Hippotion eson (Cramer, 1779) - c

Hippotion melichari Haxaire, 2001 - p

Hippotion griveaudi Carcasson, 1968 - b, c

Hippotion butleri (Saalmüller, 1884) - b, c, p, r

Rhagastis lambertoni (Clark, 1923) - a, b, c, g, h, k, o, p, q

Dans l'ensemble, la systématique adoptée ici pour cette famille repose sur les travaux de Griveaud (1959) et de VIETTE (1990), mais en tenant compte des trois sousfamilles retenues par MINET (1994) et - en règle générale - de la classification proposée par KiTching \& CADIOU (2000 : 16-19). Aux 34 taxons énumérés ci-dessus, il conviendrait d'ajouter au moins une espèce du genre Macroglossum Scopoli, 1777 (DCL : f). Il existe donc au moins 35 espèces de Sphingidae à Masoala, soit plus de la moitié des «Sphinx » actuellement connus de la Grande Île (59 espèces). 


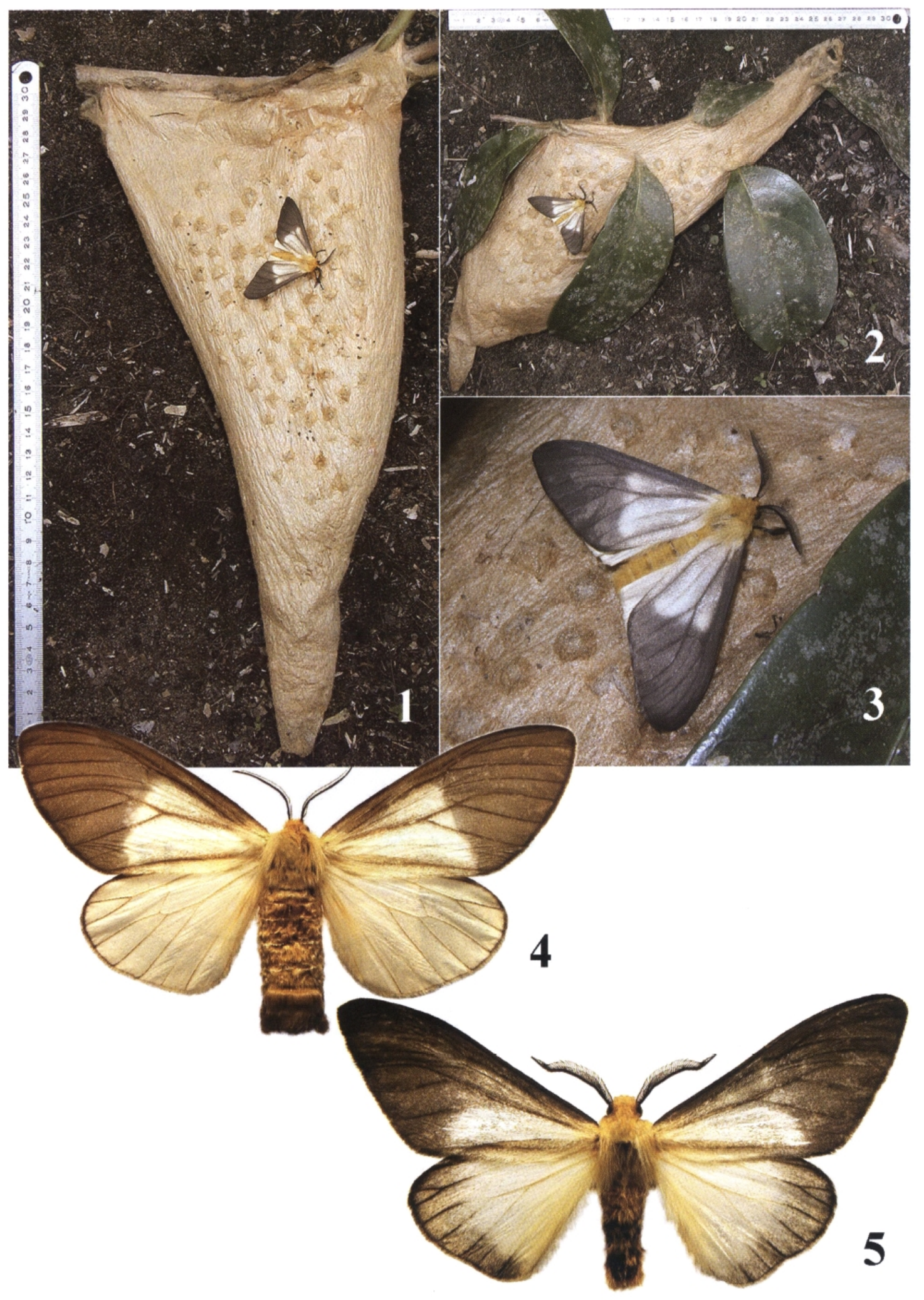



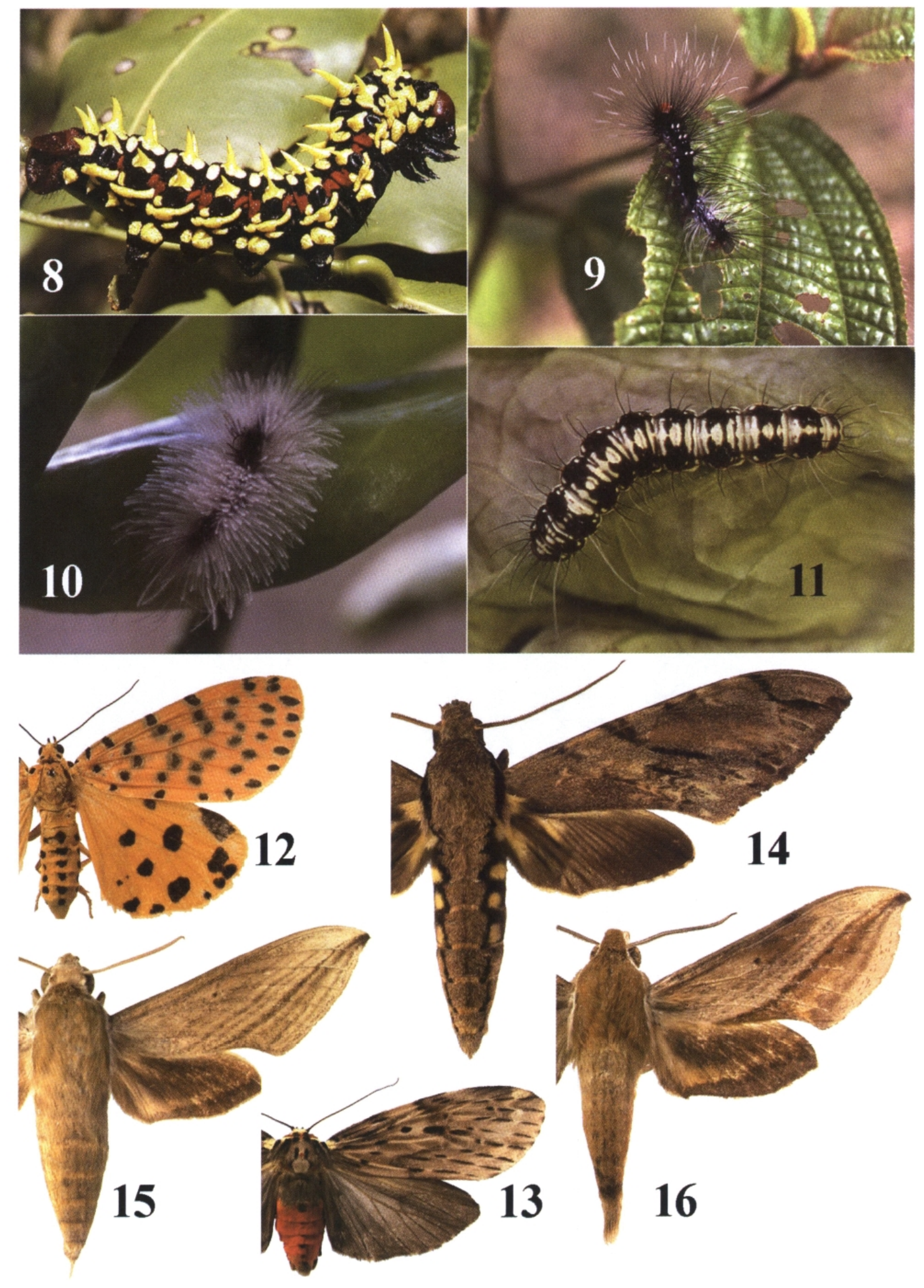


\section{LÉGENDES DES FIGURES}

Figures 1-5 - Hypsoides sp. (Notodontidae Thaumetopoeinae) [photos H.-P. Aberlenc]. 1 : nid de grande taille trouvé sur une liane au sud de Tampolo (il s'agit d'une bourse garnie de nombreux cocons). 2 : nid de même provenance, mais plus petit. 3 : mâle posé sur le nid dont il provient. 4 : femelle (envergure : $74 \mathrm{~mm}$ ) issue d'un des nids découverts au sud de Tampolo. 5 : mâle (envergure : $54 \mathrm{~mm}$ ) présentant une coloration atypique des ailes postérieures.

Figure 6-7 - 6: piège lumineux du camp de Tampolo [photo E. Faure]. 7 : David Lees (à gauche) et Joël Minet (à droite) sous la bâche protégeant ce piège [photo H.-P. Aberlenc].

Figures 8-16 - Bombycoïdes et Noctuoïdes de Masoala [photos H.-P. Aberlenc (8) et J. Minet (9-16)]. 8 : Bunaea aslauga (Saturniidae) (ex larva, envergure : $127 \mathrm{~mm}$ ), chenille trouvée à Tampolo sur Intsia bijuga (Fabaceae). 9 : chenille de Lymantriidae trouvée au nord de Tampolo, le 7 novembre 2001, sur Clidemia hirta (Melastomataceae - plante toutefois non consommée en captivité). 10 : chenille d'une autre espèce de Lymantriidae, également découverte au nord de Tampolo le 7 novembre 2001 (plante-hôte : Scaevola sericea-Goodeniaceae). 11 : Argina astrea (Arctiidae), dernier stade larvaire ( $a b$ ovo : ponte de la femelle représentée ci-après) [faute de mieux, cette chenille peut consommer de la laitue, mais en petite quantitél. 12 : Argina astrea, femelle (envergure : $40 \mathrm{~mm}$ ) provenant de Tampolo. 13 : Phryganopteryx convergens (Arctiidae), femelle (envergure : $60 \mathrm{~mm}$ ) ayant pondu de nombreux œufs ; Ambatoavo, piège lumineux UV, 16-17.xi.2001 (DCL \& JM). 14 : Xanthopan morganii praedicta (Sphingidae), mâle (envergure : $130 \mathrm{~mm}$ ) ; Tampolo, piège lumineux UV, 15.xi.2001 (HPA). 15 : Hippotion melichari (Sphingidae), mâle (envergure : $65 \mathrm{~mm}$ ) ; Tampolo, piège lumineux UV, 1.xi.2001 (HPA). 16 : Rhagastis lambertoni (Sphingidae), mâle (envergure : $59 \mathrm{~mm}$ ) ; Ambatoavo, piège lumineux UV, 1617.xi.2001 (DCL \& JM).

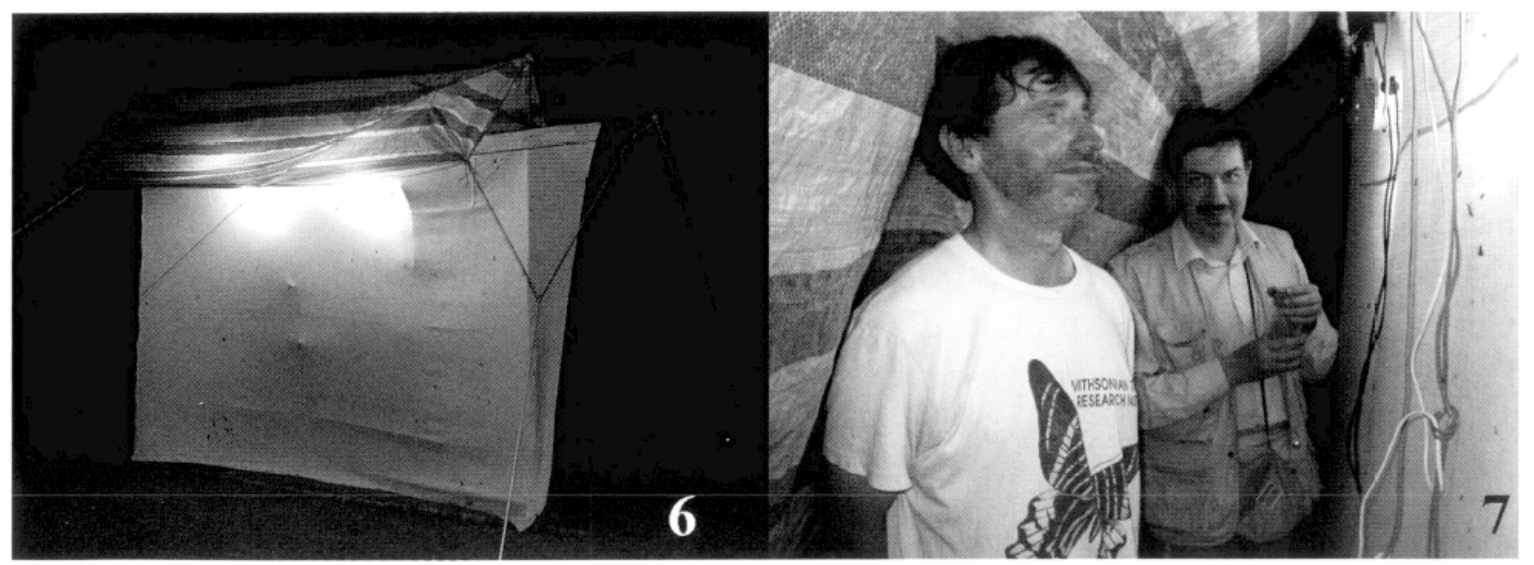




\section{EXPLICATION DES INFORMATIONS CODÉES}

a : à l'est de Hiaraka (circa 15³0'S, 4956'E), 1000 m, janvier 1968

b : idem, $1000 \mathrm{~m}$, octobre 1968

c : idem, $500 \mathrm{~m}$, octobre 1968

d : Ambatoavo (1540’S, 4959'E), $600 \mathrm{~m}$, février 1993

e : Andranobe (1540'54's, 4957'26’'E), $0 \mathrm{~m}$, février 1993

f : Andranobe : rivière Masioposo, février 1993

$\mathrm{g}$ : piste Andranobe-Be Dinta (15³8'30'S, 4958'30'E), 675 m, février 1993

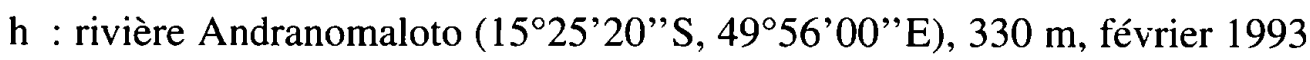

i : idem (circa 15²6' $\mathrm{S}, 4^{\circ} 57^{\prime} \mathrm{E}$ ), environ $700 \mathrm{~m}$, février 1993

j : idem (15²6’30'S, 4957'30'E), environ $900 \mathrm{~m}$, février 1993

k : Mont Mahalevona (15²6’00”S, 4957’30”E), $1220 \mathrm{~m}$, février 1993

1 : Andranobe, mars 1993

m : Cap Est (circa 151' S, 50²7’E), 0 m, janvier 1994

n : Ratsianarana (circa $15^{\circ} 28^{\prime} \mathrm{S}, 50^{\circ} 26^{\prime} \mathrm{E}$ ), $0 \mathrm{~m}$, janvier 1994 2001

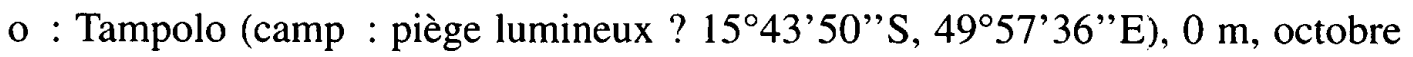

p : idem, novembre 2001

q : Ambatoavo (1540'13”S, 4958'40”E), $600 \mathrm{~m}$, novembre 2001

r : Radeau des Cimes (1542'46”S, 4958'16”E), 150 m, novembre 2001.

\section{RHOPALOCÈRES}

En ce qui concerne les Rhopalocères de Masoala, 135 espèces avaient été récemment recensées (KREMEN et al., 2001). Un travail en préparation (principal auteur : DCL) permet désormais d'avancer de nouveaux chiffres : 152 espèces pour la presqu'île Masoala et 162 pour la région de Masoala, celle-ci englobant les environs de Maroantsetra et d'Antalaha. Naturellement, cette publication tiendra compte des 65 espèces capturées ou observées au cours de la mission Radeau des Cimes 2001, c'està-dire dans la région de Tampolo-Andranobe. Nous ne mentionnerons ici que quelques espèces intéressantes à divers titres. Parmi les neuf espèces d'Hesperiidae collectées en novembre 2001, trois sont franchement nouvelles pour Masoala, à savoir Perrotia howa (Mabille, 1876), Perrotia silvestralis (Viette, 1956) et Borbo ratek (Boisduval, 1833). Cette dernière espèce n'était d'ailleurs pas rare autour de Tampolo. Pour les Papilionidae et les Pieridae, respectivement six et huit espèces ont été aperçues, voire capturées, pendant notre mission. La Piéride Belenois helcida (Boisduval, 1833) était particulièrement abondante. Au sein des Nymphalidae, trois Charaxinae ont pu être piégés en utilisant des bananes comme appâts : Euxanthe madagascariensis (Lucas, 1843) 
(EF ; CR), Charaxes cacuthis Hewitson, 1863 (CR) et Charaxes analava Ward, 1872 (CR). Précisons qu'une femelle d'E. madagascariensis a été récupérée le 19 novembre dans un piège situé sous le Radeau des Cimes, à environ $1,50 \mathrm{~m}$ du sol (EF). Le Charaxes phraortes Doubleday, 1847 - connu des environs de Maroantsetra — reste apparemment introuvable dans le Parc de Masoala. Sans doute conviendrait-il de le rechercher en pleine saison des pluies. Notons encore que le rare Heliconiinae Smerina manoro (Ward, 1871), jusqu'ici non signalé avec certitude de Masoala, a été vu et clairement identifié par l'un d'entre nous (JM) le 12 novembre 2001, sur une plage située un peu au nord de Tampolo, en lisière de forêt littorale. Enfin, parmi les dix espèces de Lycaenidae capturées dans les environs de Tampolo et d'Andranobe (en novembre), on peut souligner la présence de trois espèces du genre Saribia Butler, 1878 (Riodininae), dont $S$. ochracea Riley, 1932 (détermination : DCL ; taxon nouveau pour Masoala), et de deux Iolaus Hübner, [1819] (Lycaeninae), dont un spécimen d'I. (Trichiolaus) mermeros (Mabille, 1878) (sentier au sud de Tampolo, le 15 novembre 2001 $15^{\circ} 44^{\prime} 05^{\prime \prime} \mathrm{S}, 49^{\circ} 57^{\prime} 25^{\prime \prime} \mathrm{E}-\mathrm{JM}$ ).

Pour les Rhopalocères comme pour beaucoup d'autres Lépidoptères, la période d'octobre-novembre n'est pas la plus propice aux collectes, ce qui peut expliquer l'apparente absence de certains groupes dans la région de Tampolo (Hesperiidae Coeliadinae, par exemple). Afin d'approfondir les présentes investigations, déjà prometteuses, il faudrait pouvoir envisager de nouvelles missions dans la zone prospectée, cette fois pendant la saison des pluies... en prenant soin d'éviter les cyclones.

Remerciements. - Nous remercions les botanistes qui ont identifié les planteshôtes de diverses chenilles, notamment le Pr. Francis Hallé et le Dr Solo Rapanarivo, responsable de l'Herbier du PBZT (Parc Botanique et Zoologique de Tsimbazaza). Notre gratitude va également aux collègues collecteurs ou accompagnateurs : nous pensons naturellement aux porteurs et aux grimpeurs professionnels, mais aussi à Gianfranco Curletti (entomologiste), Thomas Haevermans (botaniste), Antoine de Maximy (cinéaste), Laurent Pyot (photographe), Hantanirina L. Rakotoarivony (entomologiste) et Sybille Unsicker (biologiste) ; à ceux qui ont organisé cette mission ? en particulier Karine Genser et Olivier Pascal (de Pro-Natura International), Messieurs Robert Rajaonarison (Directeur du Parc national de Masoala) et Michel Ranarivelo (enseignant-chercheur à l'ESPA), les collègues d'Océan Vert... ? ; à ceux qui, directement ou indirectement, nous ont permis d'y participer : Madame Olga B. Ralisoa (Pr. ? Université d'Antananarivo) et Messieurs Luc Legal (Université Paul Sabatier, Toulouse), Gilbert Rakotoarisoa (Chef du Département Faune ? PBZT) et Daniel Rakotondravony (Pr. ? Université d'Antananarivo). Nous remercions aussi vivement Monsieur Gérard Eyriès et Madame Stéphanie Gorre-Matillon, de Bayer Agro, qui ont financé la mission d'HPA et lui ont procuré un appareil photo numérique.

Enfin, nous remercions chaleureusement nos collègues Ian J. Kitching, Claire Kremen, Rodolphe Rougerie et Pierre Viette pour diverses informations, Bernard Lalanne-Cassou pour la numérisation de certaines diapositives, ainsi que nos conjoints qui ont accepté une séparation temporaire due à notre passion pour l'histoire naturelle. 


\section{SITE INTERNET}

http://www.radeau-des-cimes.org/

\section{RÉFÉRENCES BIBLIOGRAPHIQUES}

Bendib A. et Minet J., 2000 [1999]- Lithosiine main lineages and their possible interrelationships. I. Definition of new or resurrected tribes (Lepidoptera: Arctiidae). Annales de la Société entomologique de France (n. s.), 35 (3-4) : 241-263.

Borror D. J., De Long D. M. et Triplehorn C. A., 1981 - An introduction to the study of Insects. Fifth edition. Saunders College Publishing, Philadelphia, $x i+827$ p.

Desegaulx DE Nolet A., 1984 - Lépidoptères Rhopalocères, Arctiidae, Sphingidae de l'Océan Indien : Comores, Mascareignes, Seychelles. Agence de coopération culturelle et technique, Paris, $\mathrm{viii}+81 \mathrm{p}$.

Griveaud P., 1959 - Insectes. Lépidoptères Sphingidae. Faune de Madagascar, 8, 161 p.

Griveaud P., 1962 - Insectes. Lépidoptères Eupterotidae et Attacidae. Faune de Madagascar, 14, 64 p. $+12 \mathrm{pl}$.

HaXAIRE J., 2001 - Un nouveau Sphingidae du nord-est de Madagascar : Hippotion melichari (Lepidoptera Sphingidae). Lambillionea, 101 (4) : 600-604.

KIRIAKoff S. G., 1969 - Insectes. Lépidoptères Notodontidae. Faune de Madagascar, 29, 230 p. + 8 pl.

KITCHING I. J. et CADIOU J.-M., 2000 - Hawkmoths of the world: an annotated and illustrated revisionary checklist (Lepidoptera: Sphingidae). The Natural History Museum, London \& Cornell University Press, Ithaca, ix +227 p. +8 pl.

Kremen C., 2003 - The Masoala Peninsula. In : Goodman S. M. \& Benstead J. P. (éd.). The natural history of Madagascar : 1459-1466. The University of Chicago Press, Chicago.

Kremen C., Lees D., Razafimahatratra V. et Raharitsimba H., 2001 - Designing a new National Park in Madagascar: the use of biodiversity data. In : WEBER W. et al. (éd.). African rain forest ecology and conservation: 400-428. Yale University Press, New Haven \& London.

KRISTEnSEN N. P., 1998 - The non-glossatan moths. In : KRISTENSEN N. P. (éd.). Lepidoptera, moths and butterflies. Vol. 1: evolution, systematics, and biogeography (Handbuch der Zoologie [founded by Willy Kükenthal ; Fischer M. (éd.)], Band 4, Teilband 35) : 41-49. Walter de Gruyter, Berlin.

LEES D. C. et MINET J., 2003 - Lepidoptera: systematics and diversity. In : GoOdMAN S. M. \& BEnSTEAD J. P. (éd.). The natural history of Madagascar : 748-761. The University of Chicago Press, Chicago.

Migdoll I., 1988 - Field guide to the butterflies of southern Africa. New Holland Press, London, $256 \mathrm{p}$.

Minet J., 1976 - Contribution à l'étude des microlépidoptères de Madagascar [Sesiidae, Alucitidae]. Bulletin de la Société entomologique de France, 81 (1-2) : 40-43.

Minet J., 1994 - The Bombycoidea: phylogeny and higher classification (Lepidoptera: Glossata). Entomologica scandinavica, 25 (1) : 63-88.

OBERTHür C., 1923 - Lépidoptères nouveaux ou peu connus de Madagascar, d'Amérique et d'Europe. Études de Lépidoptérologie comparée, 21 (2) : 113-179+116 pl.

PAULIAN R., 1951. - Papillons communs de Madagascar. Institut de recherche scientifique, Tananarive, 91 p. +6 pl.

VIETTE P., 1956 - Nouveaux microlépidoptères de Madagascar. Bulletin de la Société zoologique de France, 81 (2-3) : 88-98.

VIETTE P., 1969 - Compte rendu de ma sixième mission entomologique à Madagascar. Lambillionea, $68(5-6): 21-34$.

VIETTE P., 1990 - Liste récapitulative des Lépidoptères hétérocères de Madagascar. A provisional check-list of the Lepidoptera Heterocera of Madagascar. Faune de Madagascar, suppl. 1, 263 p. 